\title{
Membrane fusion mediated by non-covalent binding of re-engineered cholera toxin assemblies to glycolipids
}

Sarah Wehrum $\ddagger^{a, b}$, Daniel J. Williamson $\ddagger^{c}$, Lina Siukstaite $\ddagger^{a, b}$, Thomas R. Branson $\ddagger^{c}$, Taras Sych ${ }^{a, b, d, e}$, Josef Madla,b,d, Gemma C. Wildsmith ${ }^{c}$, Wenyue Daic, James F. Ross ${ }^{c}$, Maren Thomsen ${ }^{f}$, Michael E. Webb', Winfried Römer*a,b,d and W. Bruce Turnbull*c

a. Faculty of Biology, Albert-Ludwigs-University Freiburg, Schänzlestraße 1, 79104 Freiburg, Germany. Email: winfried.roemer@bioss.uni-freiburg.de

b. Bioss - Centre for Biological Signalling Studies, Albert-Ludwigs-University Freiburg, Schänzlestraße 18, 79104 Freiburg, Germany.

c. School of Chemistry and Astbury Centre for Structural Molecular Biology, University of Leeds, Leeds LS2 9JT, UK. Email: w.b.turnbull@leeds.ac.uk

d. Freiburg Center for Interactive Materials and Bioinspired Technology (FIT), Albert-Ludwigs-University Freiburg, Georges-Köhler-Allee 105, 79110 Freiburg, Germany

e. Science for Life Laboratory, Department of Women's and Children's Health, Karolinska Institutet, 17165, Solna, Sweden

f. School of Biomedical Sciences and Astbury Centre for Structural Molecular Biology, University of Leeds, Leeds LS2 9JT, UK

†Electronic Supplementary Information (ESI) available: Supplementary figures S1-S6 and supplementary videos SV1-SV8 and experimental. See DOI: 10.1039/x0xx00000x

¥These authors contributed equally.

Membrane fusion is essential for the transport of macromolecules and viruses across membranes. While glycan-binding proteins (lectins) often initiate cellular adhesion, subsequent fusion events require additional protein machinery. No mechanism for membrane fusion arising from simply a protein binding to membrane glycolipids has been described thus far. Herein we report that a biotinylated protein derived from cholera toxin, becomes a fusogenic lectin upon crosslinking with streptavidin. This novel reengineered protein brings about hemifusion and fusion of vesicles as demonstrated by mixing of fluorescently labelled lipids between vesicles as well as content mixing of liposomes filled with fluorescently labelled dextran. Exclusion of the complex at vesicle-vesicle interfaces could also be observed indicating the formation of hemifusion diaphragms. We propose that negative membrane curvat ure, caused by binding of the cholera toxin to the membrane surface, induces formation of a fusion stalk as a result of high bending energies building up between multiple inverted membrane dimples aligned on opposing membranes at the vesicle-vesicle interface. Discovery of this fusogenic lectin complex demonstrates that new emergent properties can arise from simple changes in protein architecture and provides insights towards new mechanisms of lipid-driven fusion.

\section{Introduction}

Confinement is fundamentally important for living systems, allowing the segregation of different biochemical environments through the use of lipid bilayers. In order to maintain the integrity of the boundaries of cells or organelles, many cellular processes require membrane fusion to transport impermeable macromolecules between compartments through the exchange of trafficking vesicles. ${ }^{1-4}$ Among the most intensively studied fusion proteins are the soluble $\mathrm{N}$-ethylmaleimide-sensitive factor attachment protein receptor (SNARE) proteins. When a four helix coiled-coil bundle is formed between SNARE proteins of opposing membranes, sufficient free energy is released to pull the membranes together and induce fusion. ${ }^{3-5}$ Furthermore, extra-and intracellular fusion of pathogens with host cells is essential for infectivity. For example, enveloped viruses use transmembrane glycoproteins with short amphiphilic peptide domains to insert into the target membrane. A subsequent conformational change brings the viral and target membrane into close proximity to enable fusion. ${ }^{1,6,7}$

The mechanisms of this transition from adhesion to fusion remain a matter of ongoing investigation but are best described by the stalk hypothesis. ${ }^{1,8,9}$ In this model, fusion proceeds through a stalk intermediate in which the outer (proximal) but not the inner (distal) membrane leaflets of approaching membranes have fused. Expansion of the fusion stalk results in a hemifusion diaphragm (HD) until formation of a fusion pore in the HD completes the fusion reaction. Many details of the underlying molecular mechanisms have been addressed using simpler lipidated model systems ${ }^{10}$ including small molecules, ${ }^{11}$ coiled-coil structures, ${ }^{12-14}$ or complementary DNA ${ }^{15-17}$ or peptide nucleic acid strands. ${ }^{18}$

While some proteins are inherently fusogenic, other proteins, such as bacterial toxins, can mediate adhesion and membrane bending reminiscent of the early stages of endocytosis, ${ }^{19}$ but lack the ability to fuse membranes together. For example, cholera toxin from Vibrio cholerae is a member of a larger family of $A B_{5}$ toxins which comprise a single toxic $A$-subunit associated with a non-toxic, pentameric B-subunit (CTB). ${ }^{20}$ The A-subunit is composed of the enzymatically active A1-domain and the A2domain which protrudes through the central pore of the doughnut-shaped ring formed by the CTB pentamer (Fig. 1a)..$^{21}$ The latter is responsible for the initial adhesion to enable the toxin to enter host cells by specific binding to the high affinity ligand ( $K_{d}=10-40 \mathrm{nM}$ for a monovalent interaction) GM1 ganglioside through its branched pentasaccharide. ${ }^{22}$ The crystal structure of CTB reveals one binding site for $\mathrm{GM} 1$ per protomer, all of which are on the same face of the pentamer. ${ }^{23}$ Therefore, the lectin is pre-disposed to bind to several ligands on a single membrane, which leads to receptor clustering, ${ }^{24}$ that under certain conditions can induce phase separation of membranes, ${ }^{25}$ or the formation of tubular membrane invaginations, a prerequisite for endocytosis, as previously described for different $A B_{5}$ toxins. ${ }^{26-29}$

In this study, we sought to investigate if a simple change in lectin architecture could give rise to new emergent properties in addition to, or in place of, the membrane invagination observed upon binding to cells and GM1-functionalized synthetic membranes. ${ }^{29}$ Therefore, a strategy was developed to prepare 
complexes of CTB pentamers, in which a non-toxic $A B_{5}$ protein is modified with biotin by oxime ligation at the $\mathrm{N}$-terminus of the $\mathrm{A} 2$ peptide chain to allow complexation with streptavidin in a controlled orientation (Fig. 1b). We demonstrate that such novel biotin-modified $\mathrm{AB}_{5}$ cholera toxin-streptavidin complexes (Strep- $\left.\left(A B_{5}\right)_{n}\right)$ exhibit both crosslinking and fusogenic functions. In contrast to the aforementioned fusogenic strategies in which complementary recognition elements are lipidated and introduced into separate vesicles, ${ }^{10-18}$ the Strep- $\left(A B_{5}\right)_{n}$ is a soluble protein i.e., it is neither embedded, nor covalently attached to the membrane, but binds non-covalently to liposomes containing the GM1 ganglioside. Streptavidinmediated crosslinking of two or more $\mathrm{AB}_{5}$ complexes, allows the assemblies to bind to two opposing membranes in parallel, which is the first prerequisite for fusion. Hemifusion and fusion events were observed by transfer of fluorescently labelled lipids from one membrane to the other, and mixing of the vesicles' fluorescent content, respectively, and also leakage or rupture of liposomes. This emergent behaviour is dependent upon the formation of the multimeric Strep- $\left(A B_{5}\right)_{n}$, as the parent CTB pentamer only induces membrane invaginations. ${ }^{29}$

\section{Results}

Preparation and characterization of biotinylated $A B_{5}$ complexes

A construct for periplasmic assembly of non-toxic $A B_{5}$ analogues of cholera toxin in E. coli cells was designed in analogy to earlier work by Jobling and Holmes. ${ }^{30}$ Plasmid pSAB2.1 (Supplementary Fig. S7) allowed co-expression of CTB and a maltose-binding protein (MBP)-A2 fusion protein to enable a two-step purification strategy for isolating the $A B_{5}$ proteins. MBPcontaining proteins could first be isolated using an amylose affinity resin, before removing wild-type $E$. coli MBP from the $A B_{5}$ species by size exclusion chromatography (SEC), or by exploiting the inherent ability of CTB to bind to a nickel chelation resin. ${ }^{31}$ The resulting MBP-A2/CTB $A_{5}$ proteins were sufficiently stable to be observed by SDS-PAGE as long as the samples were not boiled prior to electrophoresis (Fig. 2a). However, extended exposure of samples to the SDS-containing loading buffer prior to analysis, led to some dissociation of $A B_{5}$ species into their MBP-A2 and CTB pentamer components.

A Tobacco Etch Virus (TEV) protease recognition site between the $\mathrm{MBP}$ and $\mathrm{A} 2$ domains was included to allow subsequent removal of the MBP domain to give an $A 2 / C T B A B_{5}$ protein. The construct used for the oxime ligation strategy was designed to leave an $\mathrm{N}$-terminal serine residue after cleavage with TEV protease (Fig. 1b). It was therefore necessary to employ a threonine to alanine mutation at the $\mathrm{N}$-terminus of the CTB sequence to prevent concomitant oxidation of the CTB protomers when exposed to periodate. ${ }^{32,33}$ Following TEV protease treatment, the $A 2 / C T B A B_{5}$ species was repurified on a nickel-chelation resin and oxidised with sodium periodate. After 5 minutes, electrospray mass spectrometry (ES-MS) confirmed complete oxidation of the terminal serine group, which was subjected to aniline-catalysed oxime ligation with oxyamine-biotin derivative biotin-1 (Fig. 1C). ES-MS analysis confirmed the formation of the biotinylated A2 peptide and also that CTB had been unaffected by the oxidation and oximation reactions (Fig. 2b, c). Similar ES-MS results (Supplementary Fig. S11) were obtained when disulfide-linked oxyamine biotin-2 (Fig. 1c) was ligated onto the oxidised $\mathrm{AB}_{5}$ protein.
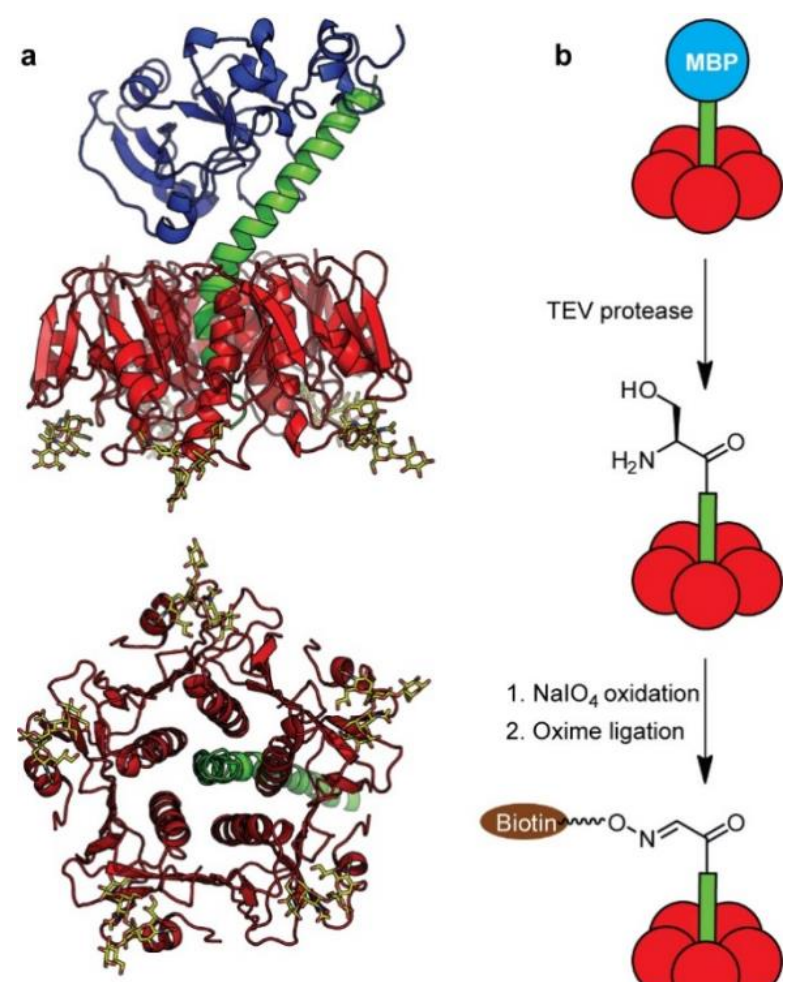

1. $\mathrm{NalO}_{4}$ oxidation

2. Oxime ligation
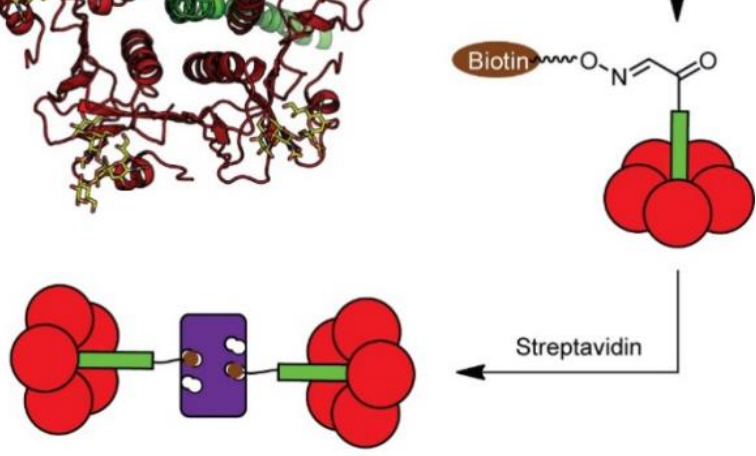

Streptavidin

C
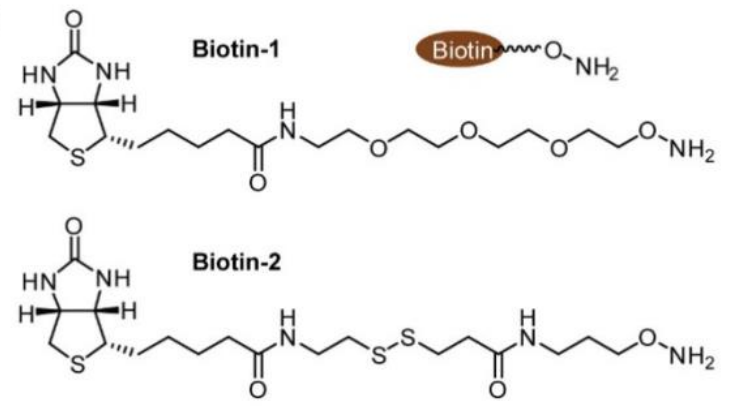

Fig. 1 (a) Model of cholera toxin based on a superposition of protein data bank files $3 \mathrm{CHB}$ and 1 XTC showing the toxic A1-subunit (blue), the A2-linker peptide (green), the pentameric B-subunit (CTB; red) and its carbohydrate ligand GM1 ganglioside (yellow); (b) A maltose-binding protein (MBP) fusion to the A2 peptide is cleaved using TEV protease to leave a serine residue that can be oxidised for oxime ligation to (c) oxyamine-biotin derivatives, prior to crosslinking with streptavidin. 


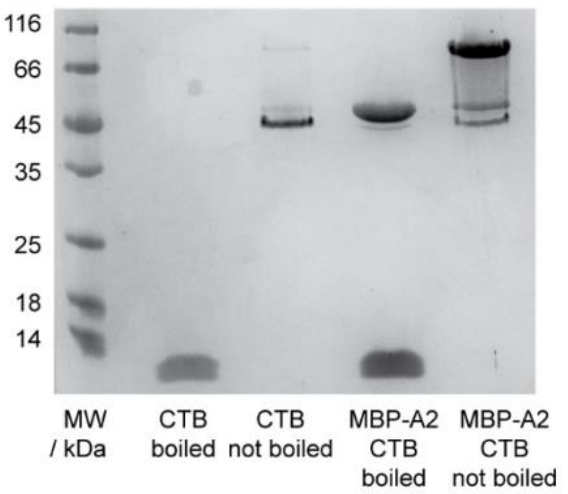

b

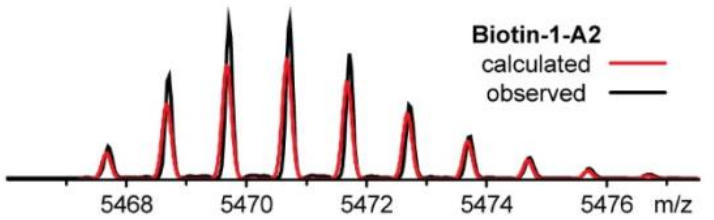

c
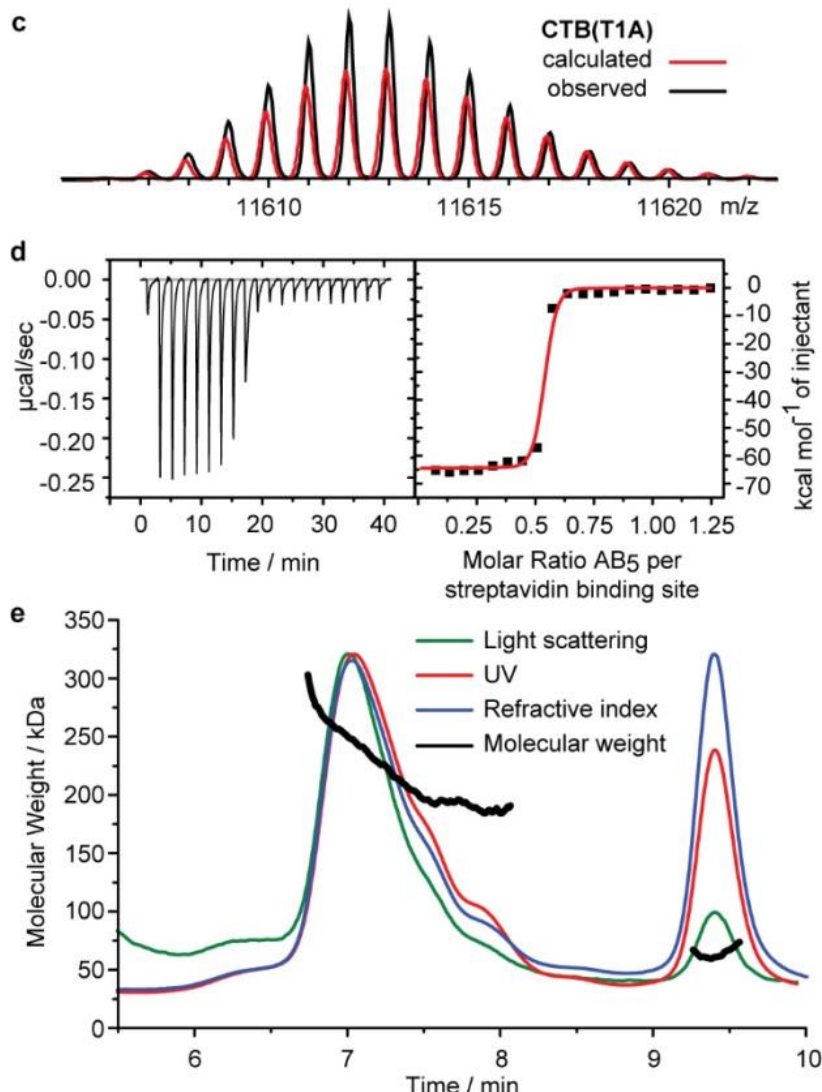

Fig. 2 Preparation of biotinylated $A B 5$ complex and its interaction with streptavidin. (a) SDS-PAGE comparison of CTB and an MBP-A2/CTB complex expressed from pSAB2.1: CTB migrates as a pentamer with apparent molecular weight of ca. $45 \mathrm{kDa}$ unless the sample is boiled prior to SDS-PAGE, in which case it migrates as a $11.5 \mathrm{kDa}$ monomer. Boiling the $A B_{5}$ complex also results in its dissociation into MBP-A2 and the CTB monomer, whereas the unboiled sample migrates as an $A B_{5}$ complex, albeit with some dissociation into the MBP-A2 and CTB pentamers; Theoretical calculated mass spectra (red) and observed deconvoluted spectra (black) for (b) the A2-peptide modified with Biotin-1, and (c) the CTB protein; (d) Isothermal titration calorimetry thermogram (left) and binding isotherm (right) showing that only $50 \%$ of streptavidin binding sites are accessible to the biotinylated $\mathrm{AB}_{5}$-complex; (e) SEC-MALS analysis of streptavidin binding showing peaks for assemblies with two to three $A B_{5}$-complexes per streptavidin (left) and unbound $A B_{5}$ protein (right)
Complexation of the biotin-1-AB 5 protein with streptavidin was studied using isothermal titration calorimetry (ITC) and size exclusion chromatography with multiple angle light scattering (SEC-MALS) analysis. Titration of biotin-1-AB ${ }_{5}$ into a solution of streptavidin (protomer concentration) indicated that the titration was complete once half of the streptavidin binding sites were filled (Fig. 2d). The binding sites of the streptavidin tetramer are arranged in pairs on opposite faces of the protein, with adjacent sites separated by ca. $20 \AA$. We thus presume that once one site is filled, the bulky protein appendage precludes easy binding to the adjacent site, and so the second biotinylated $A B_{5}$ protein preferentially binds to the opposite face of the streptavidin. ${ }^{34}$ Analysis of a mixture of biotinylated $\mathrm{AB}_{5}$ and streptavidin by SEC-MALS indicated that the complexes had masses in the range of ca. 190-250 kDa which is consistent with 2-3 $A B_{5}$ proteins per streptavidin tetramer (Fig. 2e). We would reconcile these observations by proposing that the 2:1 complexation observed by ITC initially dominates upon mixing the species, but higher complexes may also form over longer time periods in the presence of excess $A B_{5}$ protein. For simplicity, we depict 2:1 complexes in figures 1 and 6, but we note that the 3:1 complexes present will also contribute to the observed phenomena.

\section{Streptavidin- $\mathrm{AB}_{5}$ complexes crosslink GM1-coated vesicles}

The effect of the biotinylated $A_{5}$ proteins on membranes, both before and after complexation with streptavidin, were studied under well-defined conditions using giant unilamellar vesicles (GUVs). Vesicles with a lipid composition giving a liquid disordered (LD) phase (Supplementary Materials and Methods), and a defined amount of the GM1 ganglioside receptor, were mixed with the $\mathrm{AB}_{5}$ proteins/streptavidin complexes and observed by confocal microscopy. In the absence of streptavidin, incubation of biotinylated $A_{5}$ protein with vesicles resulted in the formation of tubular membrane invaginations (Supplementary Video SV1) in accordance with previous studies using CTB. ${ }^{29}$ In contrast to this behaviour, when vesicles containing 1 mol\% GM1 were incubated with $120 \mathrm{nM}$ fluorescently labelled Strep- $\left(A B_{5}\right)_{n}$, they started to adhere to each other giving rise to elongated, planar interfaces between vesicles (Supplementary Fig. S1). In some cases tubule formation could still be observed at locations on the GUVs that were distant from the crosslinked interfaces (Supplementary Video SV2). These observations were in line with our expectations that back-to-back assembly of $A_{5}$ proteins in the streptavidin complex should lead to vesicle crosslinking, as previously described by us for other carbohydrate-binding proteins that have binding sites that point in opposing directions. ${ }^{35}$

Vesicle crosslinking was dependent on complexation of the biotinylated $A B_{5}$ protein with streptavidin. Fluorescentlylabelled streptavidin complexed with $A_{5}$ proteins bearing either a PEG-linked biotin-1 or disulfide-linked biotin-2, each crosslinked GM1-functionalized vesicles (Fig. 1c; Supplementary Fig. S2). However, incubation of the disulfide-linked complex with the reducing agent dithiothreitol (DTT) resulted in a 


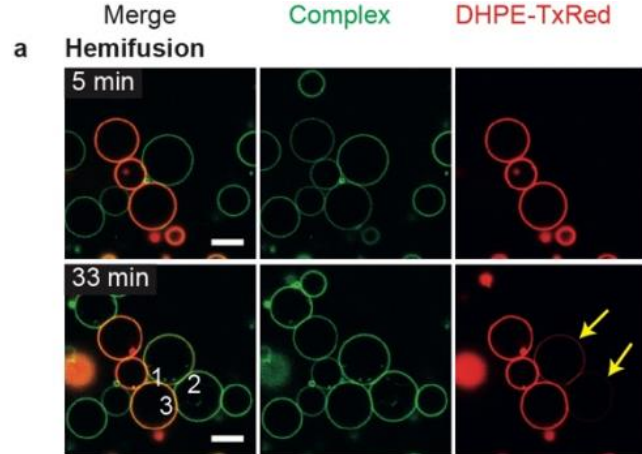

b Complex Exclusion

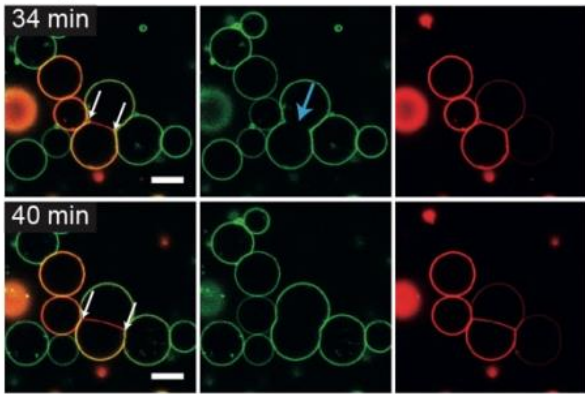

c Rupture

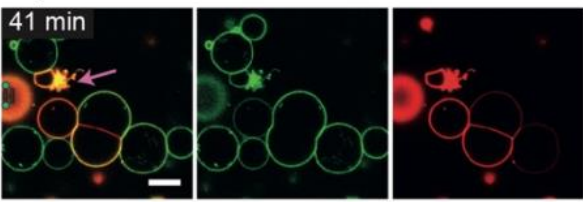

d Fusion

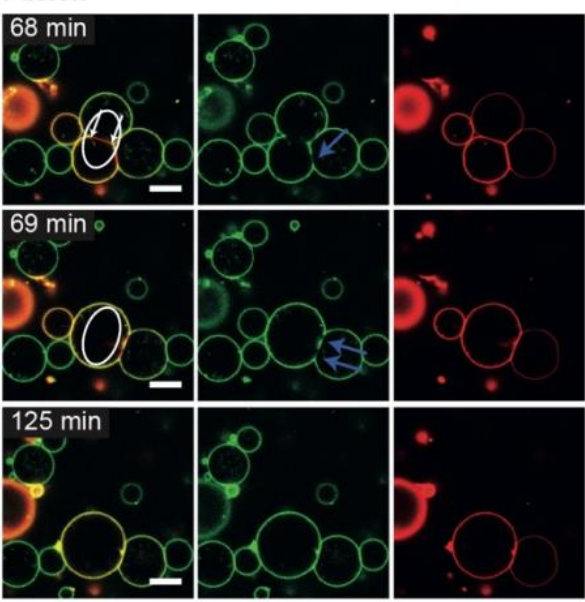

e

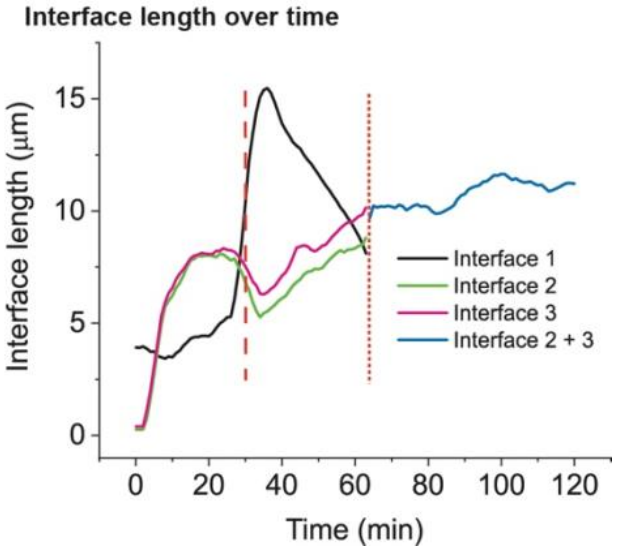

Fig. 3 Hemifusion, fusion, or vesicle rupture can be induced by Strep-( $\left(\mathrm{B}_{5}\right)_{\mathbf{n}}$ Time-series captured in $1 \mathrm{~min}$ intervals of two slightly deflated vesicle populations containing $2.5 \mathrm{~mol} \% \mathrm{GM} 1$ and either no membrane dye or $0.5 \mathrm{~mol} \%$ DHPE-TxRed (red) incubated with $100 \mathrm{nM} \mathrm{AB}$-complex ( $\mathrm{AB}_{5}$-biotin - streptavidin-AF488 (green)) for indicated time periods. (a+b) Transfer of fluorescently labelled lipids (yellow arrows) to vesicles without membrane staining indicated hemifusion of the outer membrane leaflets. (b) Crosslinking of two vesicles resulted in an elongated interface which did grow in size (distance between white arrows); yet the $A_{5}$-complex itself was excluded from the contact site (blue arrow). (c) Vesicle rupture (pink arrow) was another frequent observation. (d) Two vesicles which had already undergone hemifusion, then fuse into one (indicated by a circle). Dark blue arrows point to domains within the interface of which the $A B_{5}$-complex was excluded. (e) The dynamics of interface growth. Lengths of interfaces are derived from $2 D$ images of the GUVs' cross-sections in the focal plane displayed in the figure. Interfaces corresponding to the data presented in the graph are highlighted in panel (a) by white numbers 1,2 and 3. The dashed line marks the time point when interface 1 starts to transform into a hemifusion diaphragm. The dotted line indicates the point of fusion, after which interfaces 2 and 3 effectively become a single interface (displayed as "interface $2+3$ "). Scale bars are $10 \mu \mathrm{m}$. The full time-series can be seen in Supplementary Video SV3.

decrease ( $6 \mathrm{~h}$ treatment, Supplementary Fig. S2) or complete loss ( $27 \mathrm{~h}$ treatment) of both fluorescence and crosslinking, indicating that the fluorescently-labelled streptavidin-biotin complex was released from the $\mathrm{AB}_{5}$ protein (albeit slowly, presumably resulting from slow diffusion of the fluorescent streptavidin protein from confinement at the interface of the crosslinked vesicles). Equivalent DTT treatment of vesicles crosslinked with Strep- $\left(\mathrm{AB}_{5}\right)_{\mathrm{n}}$ based on PEG-linked biotin-1, or untreated vesicles, had no effect (Supplementary Fig. S2).

\section{Streptavidin- $A B_{5}$ complexes induce intermediate states that proceed to fusion}

In addition to crosslinking, other diverse effects were also observed, particularly at higher concentrations ( 2.5 or 5 mol\%) of the ganglioside. Observation of a mixture of fluorescently labelled and unlabelled vesicles with $100 \mathrm{nM}$ Strep- $\left(A B_{5}\right)_{n}$ over time (Fig. 3 and Supplementary Video SV3 ) revealed several additional phenomena that can be interpreted as intermediates of membrane fusion. Firstly, transfer of the membrane marker, the red-fluorescent phospholipid DHPE-TxRed, from one vesicle to an unlabelled vesicle indicated the occurrence of hemifusion of the outer membrane leaflets of approaching membranes (Fig. 3a, yellow arrows).

Furthermore, Strep- $\left(\mathrm{AB}_{5}\right)_{\mathrm{n}}$ was often excluded from the contactsite between vesicles (Fig. 3b, blue arrow), or from small areas within interfaces (Fig. 3d, dark blue arrows). This is an intriguing observation, as for other lectins with opposing binding sites, a strong accumulation within the interface was observed. ${ }^{35}$ The latter observation was attributed to restricted movement of the lectin within the planar interfaces that connect the vesicles. In contrast to the rather slow depletion of fluorescent streptavidin when the disulfide-linked complex was treated with DTT (Supplementary Fig. S2), initial exclusion of Strep-( $\left(A_{5}\right)_{n}$ in Figure $3 \mathrm{~b}$ appeared suddenly between the 33 and 34 minute frames. The planar interface continued to increase in size (Fig. $3 \mathrm{~b}$, white arrows) without accumulation of the fluorescent complex between the vesicles. We propose that hemifusion of the outer leaflets indicates the formation of a fusion stalk that can expand into a hemifusion diaphragm (HD) in which the intact inner monolayers of each vesicle, that have not been 
exposed to the complex, make contact to form a single hybrid bilayer between the vesicles.

Other frequent observations included the rupture of vesicles with complete membrane failure (Fig. $3 \mathrm{c}$ and Supplementary Video SV7) and liposome leakage without membrane lysis indicated by a slow loss of the vesicle's fluorescent dextran content (Supplementary Fig. S3). Yet, the most outstanding observation was fusion of two vesicles into one (Fig. $3 \mathrm{~d}$, white circle). In this case, fusion was preceded by a reduction of the size of the putative HD contact area from which Strep-( $\left.A B_{5}\right)_{n}$ was excluded (Fig. 3d, white arrows). Dynamic changes in the size of interfaces between the fusing vesicles and a neighbouring vesicle (interfaces 1-3 highlighted in Fig. 1a) are illustrated in Fig. 3e. While interfaces are two dimensional structures, they are usually not arranged parallel to the focal plane, therefore they appear as one dimensional structures (lines) in the fluorescence images. We thus used the length of the apparent interface as a measure for interface size, as described in the Supplementary Information. ${ }^{36}$ The data shows an initial rapid increase in length of interface 1 upon formation of the HD, and then continuous contraction to the point of fusion (dotted line). In contrast, the lengths of interfaces 2 and 3 first decrease, and then increase during this same period. This dynamic relationship of the size of HD interfaces between neighbouring vesicles probably underpins the lack of correlation between interface size and GUV radius (Supplementary Fig. S4). It is also worth noting that GUVs can rearrange in 3D which might also affect the apparent interface length.

The fusion phenomenon could also be observed by mixing of vesicle contents employing GUVs containing 5 mol\% GM1 and $200 \mathrm{nM}$ Strep-(AB$)_{n}$ (Fig. 4 and Supplementary Video SV4). Fusion of vesicles containing dextran-AF488 with vesicles containing dextran-AF647 resulted in vesicles containing both contents (Fig. 4, yellow arrows). When a larger vesicle filled with dextran-AF488 fused with a smaller vesicle filled with dextran AF647, the fluorescence intensity of the latter became more diluted and showed a lower fluorescence intensity (Fig. 4, 31 $\mathrm{min}$ ), which then increased when a second dextran-AF647 filled vesicle subsequently fused (Fig. 4, $40 \mathrm{~min}$ ) with the latter. Interestingly, there was already a slight increase of AF647

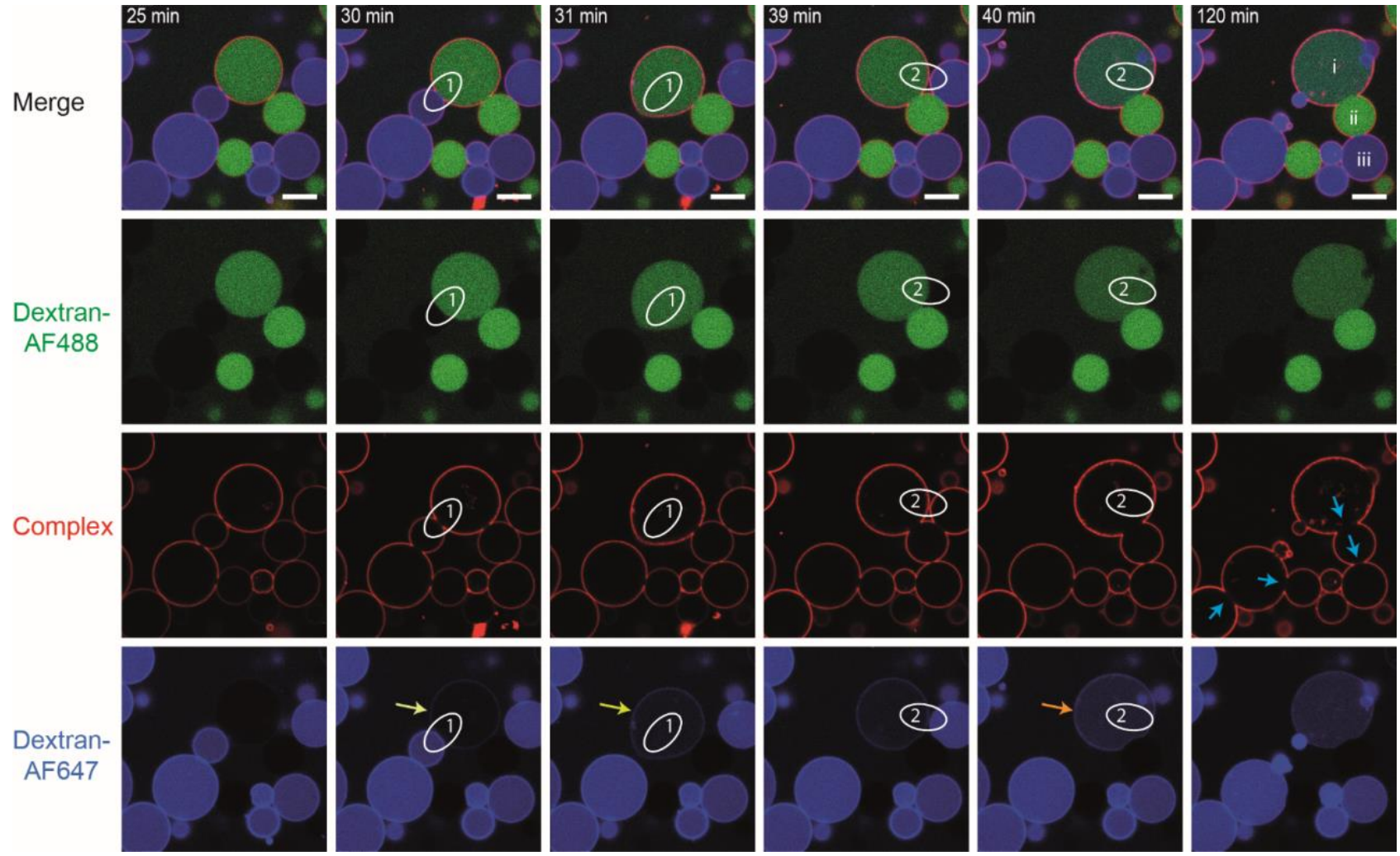

Fig. 4 Complete fusion but not crosslinking results in content mixing of vesicles incubated Strep-(AB $\left.)_{5}\right)_{n}$ Time-series captured in 1 min intervals of two slightly deflated vesicle populations ( 5 mol\% GM1 without membrane staining) filled with either dextran-AF488 (green) or dextran-647 (blue) incubated with $200 \mathrm{nM} A \mathrm{~B}_{5}-\mathrm{biotin}-$ streptavidin-AF555 (red) complex. 30 min after $A B_{5}$ complex incubation a slight increase of blue fluorescence was observed for an otherwise green fluorescent vesicle (light yellow arrow), which could indicate the opening of a fusion pore. Indeed, only one minute later, two vesicles fuse into one and the blue fluorescence intensity did further increase (yellow arrow). At 40 min the resulting vesicle of fusion event 1 fused a second time with another dextran-647 containing vesicle which further accelerated the blue fluorescence (orange arrow). Other vesicles showing significant crosslinking, even with the exclusion of Strep-(AB $)_{n}$ (blue arrows), did not show any signs of fusion or the formation of fusion pores, as contents remained completely separate (for example vesicle $\mathrm{i}$, ii, an d iii). Scale bars are $10 \mathrm{\mu m}$. The full time-series can be seen in Supplementary Video SV4. 

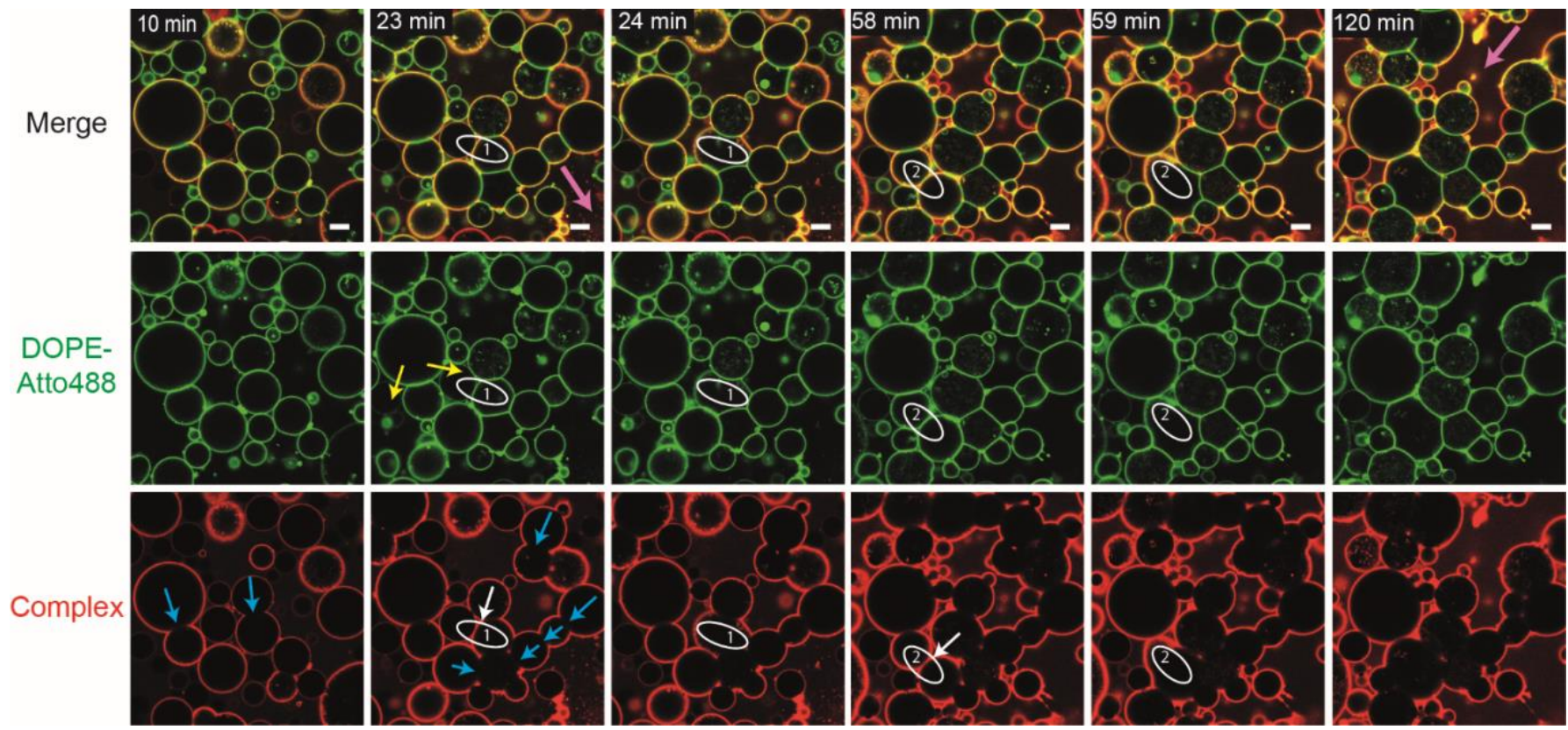

Fig. 5 Vesicle fusion does not necessarily follow nor require extensive vesicle crosslinking with complex exclusion. Time-series captured in 1 min intervals of two slightly deflated vesicle populations containing 5 mol\% GM1 and either no membrane dye or 0.5 mol\% DOPE-Atto488 (green) incubated with 200 nM AB5-biotin - streptavidin-AF555 (red) complex for indicated time periods. Blue arrows indicate the appearance of first interfaces where the $\mathrm{AB}_{5}$ complex became excluded. White arrows, on the other hand, point at contact areas,

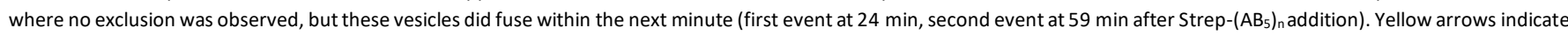
the transfer of membrane dye indicating hemifusion. The pink arrows at 23 and $120 \mathrm{~min}$ are pointing at vesicles, which did rupture towards in the course of the time-lapse. Scale bars are $10 \mu \mathrm{m}$. The full time-series can be seen in Supplementary Video SV5.

fluorescence within the AF488 vesicle some time before fusion (Fig.4, $30 \mathrm{~min}$ ), which could indicate that a small fusion pore had already formed. The accumulation of dextran-AF647 at the membrane can most likely be attributed to the properties of the fluorescent polysaccharide, as it was observed for all vesicles. Nonetheless, these experiments also provide proof that the membrane barrier was still intact for the majority of crosslinked vesicles that showed exclusion of Strep- $\left(A B_{5}\right)_{n}$ from the interface (Fig. 4, blue arrows), as no mixing of those vesicles'contents was observed (Fig. 4, 120 min, vesicles i, ii, iii). This observation is also consistent with hemifusion leading to formation of an HD between the vesicles.

Our experiments indicate that fusion is not necessarily preceded by exclusion of Strep-( $\left(\mathrm{B}_{5}\right)_{n}$ from an elongated interface between vesicles. While this process was observed in Fig. 3 and fusion event 1 of Fig. 4, there was no preceding exclusion of the complex in fusion event 2 . Similarly, the two fusion events depicted in Fig. 5 (Supplementary Video SV5) do not show elongated interfaces or complex exclusion before fusion. In this case, hemifusion could be observed for fusion event 1 by the transfer of the fluorescent phospholipid DOPEAtto488 (Fig. 5, yellow arrows). However, both vesicles involved in fusion event 2 contained the membrane dye and accordingly there could not be any indication of whether or not hemifusion did occur. In general, most interfaces of this time-lapse did show complex exclusion (Fig. 5, blue arrows), indicating hemifusion, yet without resulting in complete fusion.

Data recorded under the conditions used for Fig. 3 were used to quantify the fraction of GUV interfaces that undergo hemifusion and fusion, and data recorded for Figs. 3 and 5 were used to quantify HD formation as a function of GM1 molar percentage
(Fig. 6). While interface size was found to be independent of GM1 concentration (Supplementary Fig. S5), the number of interfaces per GUV increased with the percentage of GM1 (Supplementary Fig. S6), therefore, for consistency, the data in Fig. 6 are displayed as a fraction of interfaces rather than a
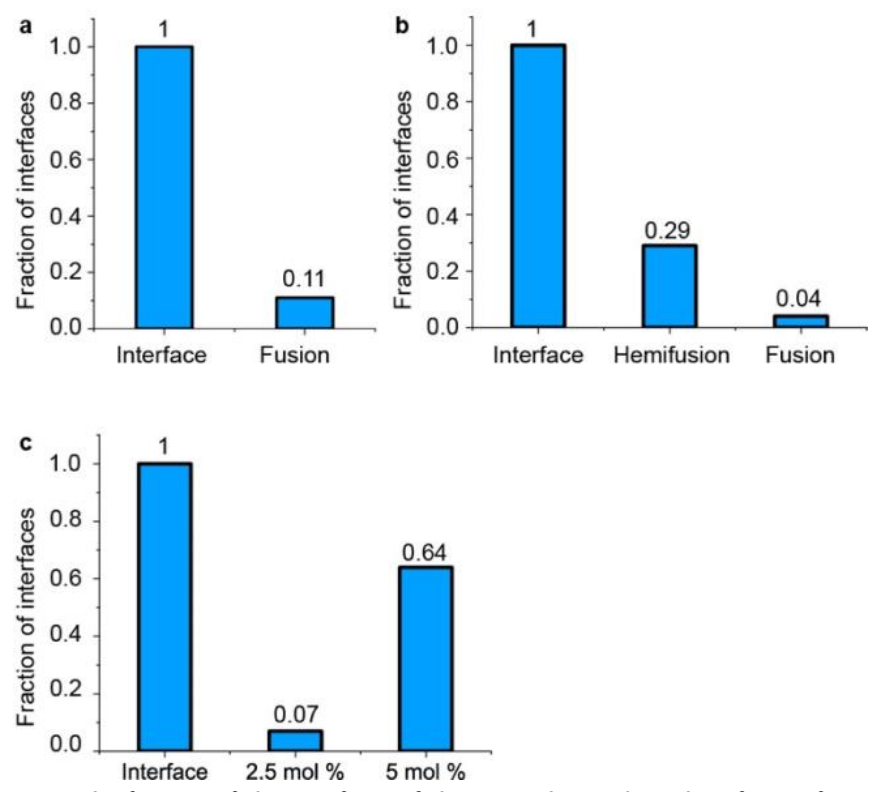

Fig. 6 The fraction of the interfaces of the GUVs that undergo hemifusion, fusion events, or form diaphragms. Data for panels (a) and (b) were extracted from the same dataset comprising 349 GUVs (2.5\% GM1; representative image: Fig. 3). (a) Eleven percent of the 205 GUV-GUV interfaces in this dataset underwent fusion. (b) Ninetyeight of the interfaces in this dataset were between labelled and unlabelled GUVs, $29 \%$ of which were observed to undergo hemifusion and $13 \%$ of these $(4 \%$ of the total)proceeded to full fusion. (c) Interfaces that transform into hemifusion diaphragms for 2.5 mol\% and 5 mol\% GM1 (representative images: Fig. 3 and Fig. 5, respectively). 
fraction of GUVs. A sample of 349 GUVs (comprising 2.5\% GM1) formed 205 interfaces with their neighbours, and $11 \%$ of such interfaces underwent fusion (Fig. 6a). Ninety-eight of these interfaces were between pairs of labelled and unlabelled vesicles, where it was also possible to quantify numbers of hemifusion events arising from transfer of labelled lipids (Fig. $6 b)$. Hemifusion was detected for $29 \%$ of interfaces between labelled and unlabelled GUVs, and $13 \%$ of these hemi-fused vesicles (i.e. $4 \%$ of the total) went on to undergo full fusion. Hemifusion can alternatively result in either vesicle collapse: of the 349 GUVs studied, 62 vesicles (18\%) exploded/collapsed. Hemifusion Diaphragm formation was found to be very dependent on GM1 concentration: only 7\% of GUV interfaces underwent $\mathrm{HD}$ formation when $2.5 \% \mathrm{GM} 1$ was used, but this rose to $64 \%$ of interfaces for GUVs with $5 \%$ GM1.

Variation in membrane elasticity, e.g. arising from osmotic effects or lipid composition, also influences the outcome of the fusion experiments. For example, more vesicle rupture was observed when the inner and outer solution were osmotically matched (Supplementary Video SV7); on the other hand, slightly deflated GUVs (10 mOsm difference) were more likely to engage in hemifusion or fusion when incubated with Strep$\left(A B_{5}\right)_{n}$ (Figs. 3-5). In all experiments described thus far, the lipid composition was designed to give liquid disordered (Ld) membranes. However, when $200 \mathrm{nM}$ of Strep-( $\left(\mathrm{B}_{5}\right)_{\mathrm{n}}$ was applied to GUVs constituted of the lipid bilayer of the rigid liquid-ordered (Lo) phase, tubule formation arising from negative membrane curvature was abolished. Lo GUVs still displayed crosslinking and HD formation, but no fusion, nor transfer of fluorescent lipid from labelled to unlabelled vesicles was observed during the 90 minute timescale of the experiment (Supplementary Video SV8). Lipid diffusion is expected to be about 10 -fold slower in Lo membranes, ${ }^{37}$ but the lack of labelled lipid transfer was perhaps surprising, as HD formation would be the result of hemifusion. However, it is notable that diffusion of fluorescent lipids between the hemifused vesicles in Fig. 3 (supplementary video SV3) slows considerably upon formation of the HD. It is possible that Strep-( $\left(\mathrm{AB}_{5}\right)_{n}$ complex bound at the edge of HD gives rise to a 'restricted hemifusion' phenotype observed in other fusogenic systems, ${ }^{38}$ and this further restricts inter-vesicle lipid diffusion.

\section{Discussion}

\section{Potential mechanisms for CTB-mediated membrane fusion}

Unlike other multimeric lectins, Strep- $\left(A B_{5}\right)_{n}$ is able to induce not just association but also fusion and rupture of GM1functionalized vesicles. How then does this complex of glycanbinding proteins cause these observed phenomena? As briefly

\section{a Crosslinking}

b "Inverted" membrane dimples
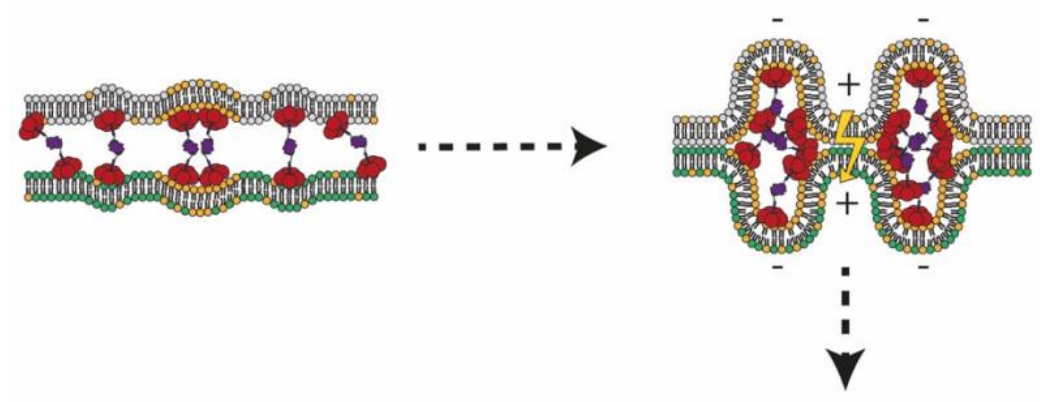

\section{e Fusion}

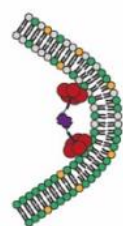

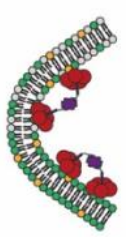
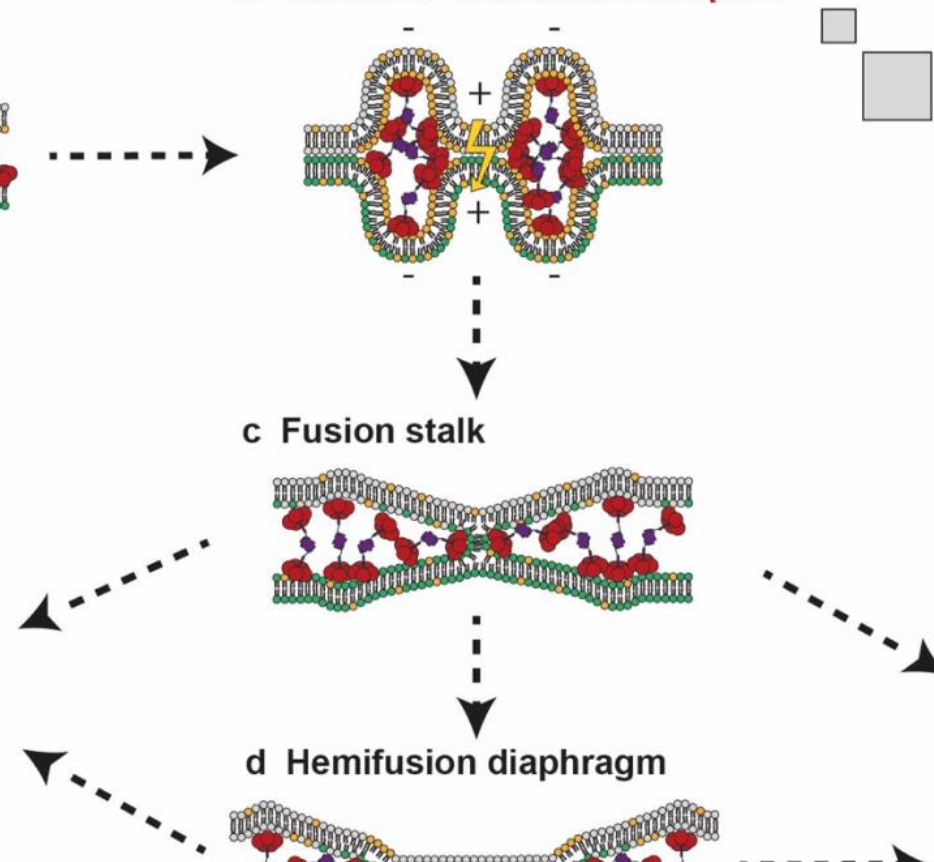

c Fusion stalk

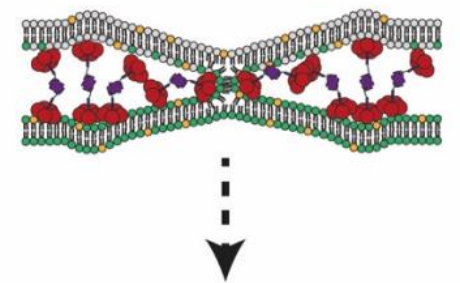

d Hemifusion diaphragm
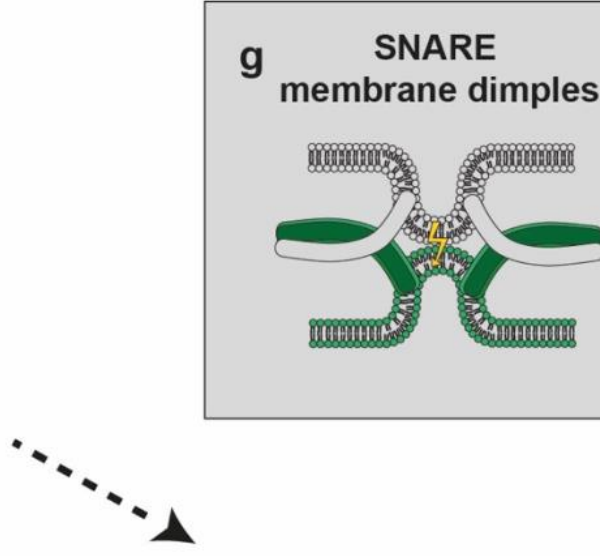

f Rupture

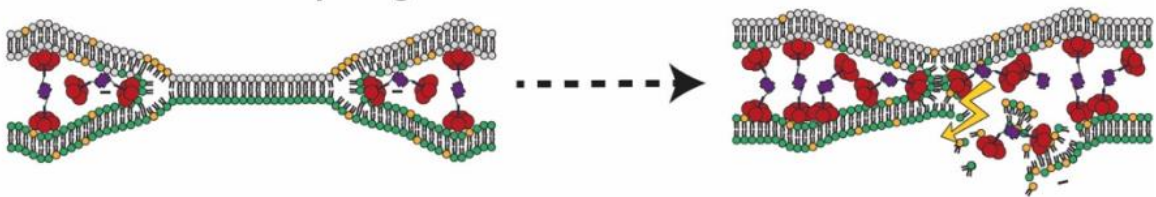

Fig. 7 "Inverted" fusion stalk hypothesis. Schematic presentation of $A_{5}$ (red)-biotin - streptavidin (purple) complexes binding to lipid bilayers containing phospholipids (white lipids), GM1 gangliosides (orange lipids) and fluorescently labelled phospholipids (green lipids) describing the possible intermediates of membrane fusion. (a) In addition to membrane crosslinking, the complex could induce negative membrane curvature at multiple sites aligned on the opposing membranes possibly leading to (b) the formation of a cluster of multiple "inverted" membrane dimples with negative membrane curvature generating positive membrane dimples on opposing contacting membranes with high bending energies (illustrated as yellow lightning) in-between which could be released by (c) the formation of a fusion stalk which could directly collapse into (e) a fusion pore or radially expand into (d) a hemifusion diaphragm (HD) from which the complex would become excluded. The negative membrane curvature at the HD rim could potentially be stabilized by Strep-( $\left.A B_{5}\right)_{n}$. until (e) opening of a fusion pore would complete fusion of the two vesicles. Alternatively, pore formation outside the fusion stalk or hemifusion diaphragm might result in (f) vesicle rupture or leakage. (g) Schematic presentation of the outward budded membrane dimples generated by SNARE in duced fusion. 
discussed in the introduction, the stalk model of membrane fusion describes the strong bending of two membranes into an hourglass shape in order to make a connection without the exposure of the hydrophobic lipid tails to water. ${ }^{8,9}$ Therefore, it has been proposed that a critical step of fusion is the local bending of membrane bilayers by fusion proteins into dimples that point towards the adjacent membrane (Fig. 7g). ${ }^{38}$ Since such bending occurs at length scales below the diffraction limit we cannot observe such changes in our data. Nevertheless, it is well established that GM1 binding by CTB results in glycolipid clustering, and leads to negative membrane curvature and invaginations (Fig. 7a), ${ }^{39}$ in part as CTB applies a downward force to the membrane through protruding alpha helices in the middle of the protein. ${ }^{40}$ That is where we think the novel architecture of Strep-( $\left.\mathrm{AB}_{5}\right)_{n}$ comes into play: due to the linkage of two to three CTB molecules which are "pushing" into opposite directions, a cluster of several membrane dimples with negative curvature could give rise to positive membrane dimples in-between, which could align opposing membranes within contact distance (Fig. 7b). Accordingly, the critical step of local bending still applies as strong bending energies that would build up at the rim of the inward bud and tip of the outside bud, would be released by the formation of a fusion stalk (Fig. 7c).

Formation of a fusion stalk accounts for the observed transfer of fluorescent lipids between adjacent vesicles and also provides an explanation for the frequently observed exclusion of Strep- $\left(A B_{5}\right)_{n}$ from the interface between vesicles. Radial expansion of the stalk would lead to a hemifusion diaphragm (HD) in which both inner membrane leaflets of the hemifused vesicles come together to create a single bilayer (Fig. 7d). HDs in $\mu \mathrm{m}$ size have been observed previously in GUV systems, ${ }^{41}$ and also during vacuolar lysosome fusion. ${ }^{42}$ It has been proposed that HD formation occurs in cases where tension of the outer leaflet of a vesicle is greater than that of the inner leaflet. ${ }^{43}$ Formation of a fusion stalk provides a mechanism to rebalance membrane tension by allowing retraction of the outer leaflet from the boundary between vesicles, which in turn leads to $H D$ expansion. ${ }^{43}$ It is possible that Strep- $\left(A B_{5}\right)_{n}$ binding to $G M 1$ may increase tension in the outer leaflet, for example by ordering lipids, ${ }^{44}$ and release of this tension drives HD formation. This would be in line with our data in Fig. $6 \mathrm{c}$ which shows that increasing the GM1 concentration, and thus GM1 clustering in the membrane, leads to a substantial increase in HD formation. Switching from Ld to Lo vesicles did not prevent HD formation. It is known that Lo GUVs do not display the large tubular invaginations upon binding to CTB that are seen for Ld GUVs. ${ }^{29}$ However, that does not preclude the possibility that smaller scale membrane bending could still occur for Lo GUVs, unobserved, below the diffraction limit of our fluorescence microscopy experiments. Indeed, membrane bending is presumably required for the hemifusion process leading to the HD. As full fusion was not observed in these experiments, Loderived hemifusion diaphragms appear to be more stable than those derived from Ld membranes, which is in line with other reports that Lo vesicles are less prone to membrane fusion. ${ }^{45}$ Irrespective of whether fusion occurs by direct collapse of the fusion stalk, ${ }^{38}$ or rupture the bilayer following formation of an
$H D, 46,47$ the process necessitates the formation of a pore in the membrane (Fig. 7e). Vesicle rupture and leakage also require pore formation, but on a portion of the membrane that does not lie at the interface between two connected vesicles. We can only speculate on the forces introduced to the membrane by Strep- $\left(A B_{5}\right)_{n}$ that could cause membrane rupture. Simunovic and co-workers have recently presented a mechanism called friction-driven scission to describe how protein scaffolds can build local membrane tension until tubular membranes undergo scission through lysis when the tube gets elongated. ${ }^{46}$ The dynamic expansion and contraction of HDs seen in Fig. 3e and Video SV3 indicates that there are significant forces acting on our GUVs and any motions will presumably be subject to friction caused by inter-GUV crosslinking by Strep- $\left(A B_{5}\right)_{n}$. Thus, it is possible that observed membrane rupture may be subject to an analogous type of friction-driven scission. While it is beyond the scope of our present work to prove conclusively the precise mechanism by which fusion is induced by Strep- $\left(A B_{5}\right)_{n}$, our data provide a new perspective on the established fusion hypotheses and support a novel strategy for fusion, involving induction of multiple aligned membrane invaginations by a glycosphingolipid-binding protein

\section{Conclusions}

We have shown that site-specific biotinylation of an $A_{5}$ bacterial toxin permits the assembly of multimeric complexes with streptavidin which exhibit new fusogenic functions. While all five binding sites for the ganglioside GM1 of the СTB pentamer face in one direction, leading to receptor clustering and negative membrane curvature, the back-to-back assembly of the complex allows for additional crosslinking of two GM1functionalized membranes resulting in vesicle clusters with planar interfaces. In contrast to previous studies in which avidin proteins have been used to crosslink biotinylated vesicles, ${ }^{49,50}$ the Strep-( $\left(B_{5}\right)_{n}$ complex was found to induce hemifusion indicated by fluorescently-labelled lipid exchange between vesicles and complete fusion of two vesicles into one with merging of the fluorescently labelled liposome contents. Exclusion of the Strep- $\left(A B_{5}\right)_{n}$ complex from the GUV-GUV interface was also frequently observed, indicating formation of a hemifusion diaphragm. HD formation increased at higher concentrations of GM1. We propose that when clusters of Strep- $\left(\mathrm{AB}_{5}\right)_{n}$ crosslink membranes, CTB-induced "inverted" membrane dimples, with very high bending energies, become aligned and upon contact between opposing membranes there is formation of a fusion stalk. Subsequent expansion of the stalk into a HD and opening of a fusion pore could complete the fusion process. This mechanism presents a different fusogenic strategy compared to the dimples budding out of a membrane by e.g. SNARE proteins or viral peptides that induce membrane fusion. This work has allowed us to identify a synthetic glycanbinding protein with fusogenic properties that acts by simply binding to a membrane component and does not require its own incorporation into the membrane. The results provide a new insight into the established hypotheses of membrane 
fusion, which is an indispensable requirement for many cellular processes but also for applications like drug delivery.

\section{Conflicts of interest}

There are no conflicts to declare.

\section{Acknowledgements}

This work has been supported in the framework of the EU ERASynBio project SynGlycTis by the German Federal Ministry of Education and Research (BMBF; FKZ 031A464) and UK Biotechnology and Biological Sciences Research Council (BB/M005666/1; BB/L015056/1); the Ministry of Science, Research and the Arts of Baden-Württemberg ( $\mathrm{Az}$ : 33-7532.20); the Excellence Initiative of the German Research Foundation [EXC 294]; the German Research Foundation grants RTG 2202 and IRTG 1642; the UK Engineering and Physical Sciences Research Council (EP/G043302/1) and The Wellcome Trust (089308/Z/09/Z; 094232/Z/10/Z). This research has received funding from the European Union's Horizon 2020 research and innovation programme under the Marie Skłodowska-Curie grant agreement numbers 708051 (individual fellowship for M.T.) and 814029 (synBIOcarb ITN). T.S. acknowledges the support of the Franco-German University (programs 'Polymer Sciences' and 'Cotutelle de these'), the College Doctoral Europeen (PDI) and the International Graduate Academy (University of Freiburg).

\section{References}

1 R. Jahn and T. C. Südhof, Annu. Rev. Biochem., 1999, 68, 863911.

2 R. Jahn, T. Lang and T. C. Südhof, Cell, 2003, 112, 519-533.

3 H. Robson Marsden, I. Tomatsu and A. Kros, Chem. Soc. Rev., 2011, 40, 1572-1585.

4 R. Jahn and R. H. Scheller, Nat. Rev. Mol. Cell Biol., 2006, 7, 631-643.

5 T. C. Südhof and J. E. Rothman, Science, 2009, 323, 474-477.

6 L. J. Earp, S. E. Delos, H. E. Park and J. M. White, Curr. Top. Microbiol. Immunol., 2005, 285, 25-66.

7 J. M. White, S. E. Delos, M. Brecher and K. Schornberg, Crit. Rev. Biochem. Mol. Biol., 2008, 43, 189-219.

8 V. S. Markin, M. M. Kozlov and V. L. Borovjagin, Gen. Physiol. Biophys., 1984, 3, 361-377.

9 V. S. Markin and J. P. Albanesi, Biophys. J., 2002, 82, 693-712.

10 M. Ma and D. Bong, Acc. Chem. Res., 2013, 46, 2988-2997.

11 M. Ma, Y. Gong and D. Bong, J. Am. Chem. Soc., 2009, 131, 16919-16926.

12 H. Robson Marsden, N. A. Elbers, P. H. H. Bomans, N. A. J. M. Sommerdijk and A. Kros, Angew. Chem. Int. Ed., 2009, 48, 2330-2333.

13 A. Kashiwada, M. Tsuboi, N. Takamura, E. Brandenburg, K. Matsuda and B. Koksch, Chem. Eur. J., 2011, 17, 6179-6186.

14 G. A. Daudey, H. R. Zope, J. Voskuhl, A. Kros and A. L. Boyle, Langmuir, 2017, 33, 12443-12452.

15 G. Stengel, R. Zahn and F. Höök, J. Am. Chem. Soc., 2007, 129, 9584-9585.

16 Y. H. M. Chan, B. van Lengerich and S. G. Boxer, Proc. Natl. Acad. Sci. U.S.A., 2009, 106, 979-984.
17 P. M. G. Löffler, O. Ries, A. Rabe, A. H. Okholm, R. P. Thomsen, J. Kjems and S. Vogel, Angew. Chem. Int. Ed., 2017, 56, 1322813231

18 M. Sadek, D. Berndt, D. Milovanovic, R. Jahn and U. Diederichsen, ChemBioChem, 2016, 17, 479-485.

19 J. Madl, S. Villringer and W. Römer, in Chemical and Synthetic Approaches in Membrane Biology, ed. A. Shukla, Humana Press, New York, NY, 2016, pp. 17-23.

20 T. Beddoe, A. W. Paton, J. Le Nours, J. Rossjohn and J. C. Paton, Trends Biochem. Sci., 2010, 35, 411-418.

21 R.-G. Zhang, D. L. Scott, M. L. Westbrook, S. Nance, B. D. Spangler, G. G. Shipley and E. M. Westbrook, J. Mol. Biol., 1995, 251, 563-573.

22 W. B. Turnbull, B. L. Precious and S. W. Homans, J. Am. Chem. Soc., 2004, 126, 1047-1054.

23 E. A. Merritt, P. Kuhn, S. Sarfaty, J. L. Erbe, R. K. Holmes and W. G. J. Hol, J. Mol. Biol., 1998, 282, 1043-1059.

24 J. Shi, T. Yang, S. Kataoka, Y. Zhang, A. J. Diaz and P. S. Cremer, J. Am. Chem. Soc., 2007, 129, 5954-5961.

25 A. T. Hammond, F. A. Heberle, T. Baumgart, D. Holowka, B. Baird and G. W. Feigenson, Proc. Natl. Acad. Sci. USA, 2005, 102, 6320-6325.

26 R. Montesano, J. Roth, A. Robert and L. Orci, Nature, 1982, 296, 651-653.

27 M. L. Torgersen, G. Skretting, B. van Deurs and K. Sandvig, J. Cell Sci., 2001, 114, 3737-3747.

28 W. Römer, L. Berland, V. Chambon, K. Gaus, B. Windschiegl, D. Tenza, M. R. E. Aly, V. Fraisier, J.-C. Florent, D. Perrais, C. Lamaze, G. Raposo, C. Steinem, P. Sens, P. Bassereau and L. Johannes, Nature, 2007, 450, 670-675.

29 H. Ewers, W. Römer, A. E. Smith, K. Bacia, S. Dmitrieff, W. Chai, R. Mancini, J. Kartenbeck, V. Chambon, L. Berland, A. Oppenheim, G. Schwarzmann, T. Feizi, P. Schwille, P. Sens, A. Helenius and L. Johannes, Nat. Cell Biol., 2009, 12, 11.

30 M. G. Jobling and R. K. Holmes, Infect. Immun., 1992, 60, 49154924.

31 M. T. Dertzbaugh and L. M. Cox, Protein Eng. Des. Sel., 1998, 11, 577-581.

32 J. Chen, W. Zeng, R. Offord and K. Rose, Bioconjug. Chem., 2003, 14, 614-618.

33 T. R. Branson, T. E. McAllister, J. Garcia-Hartjes, M. A. Fascione, J. F. Ross, S. L. Warriner, T. Wennekes, H. Zuilhof and W. B. Turnbull, Angew. Chem. Int. Ed., 2014, 53, 8323-8327.

34 M. Fairhead, D. Krndija, E. D. Lowe and M. Howarth, J. Mol. Biol., 2014, 426, 199-214.

35 S. Villringer, J. Madl, T. Sych, C. Manner, A. Imberty and W. Römer, Sci. Rep., 2018, 8, 1932-1932.

36 T. Sych, T. Schubert, R. Vauchelles, J. Madl, R. Omidvar, R. Thuenauer, L. Richert, Y. Mély and W. Römer, Bioinformatics, 2019, 35, 2340-2342

37 N. Kahya, P. Schwille Mol. Membrane Biol., 2006, 23, 29-39.

38 L. V. Chernomordik and M. M. Kozlov, Nat. Struct. Mol. Biol., 2008, 15, 675-683.

39 A. Maarouf Kabbani, K. Raghunathan, W. I. Lencer, A. K. Kenworthy, C. V. Kelly, Proc. Natl. Acad. Sci. USA, 2020, 117, 14978-14986.

40 W. Pezeshkian, L. J. Nåbo and J. H. Ipsen, FEBS Open Bio, 2017, 7, 1638-1645.

41 J. Nikolaus, M. Stöckl, D. Langosch, R. Volkmer and A Herrmann, Biophys. J., 2010, 98, 1192-1199.

42 S. Mattie, E. K. McNally, M. A. Karim, H. Vali and C. L. Brett, Mol. Biol. Cell, 2017, 28, 309-321.

43 J. M. Warner and B. O'Shaughnessy, Phys. Rev. Lett., 2012 108, 1-5.

44 K. Raghunathan, T. H. Wong, D. J. Chinnapen, W. I. Lencer, M. G. Jobling and A. K. Kenworthy, Biophys. J., 2016, 111, 25472550. 
45 S.-T. Yang, V. Kiessling, J. A. Simmons, J. M. White and L. K. Tamm, Nature Chem. Biol., 2015, 11, 424-431.

46 M. M. Kozlov, S. L. Leikin, L. V. Chernomordik, V. S. Markin and Y. A. Chizmadzhev, Eur. Biophys. J., 1989, 17, 121-129.

47 Y. Kozlovsky, L. V. Chernomordik and M. M. Kozlov, Biophys. J., 2002, 83, 2634-2651.

48 M. Simunovic, J. B. Manneville, H. F. Renard, E. Evergren, K. Raghunathan, D. Bhatia, A. K. Kenworthy, G. A. Voth, J. Prost, H. T. McMahon, L. Johannes, P. Bassereau and A. Callan-Jones, Cell, 2017, 170, 172-184.e111.

49 S. Chiruvolu, S. Walker, J. Israelachvili, F. J. Schmitt, D. Leckband and J. A. Zasadzinski, Science, 1994, 264, 1753-1756.

50 D. A. Noppl-Simson and D. Needham, Biophys. J., 1996, 70, 1391-1401. 